\title{
Transcranial magnetic stimulation to right parietal cortex modifies the attentional blink
}

Received: 15 December 2002 / Accepted: 18 August 2003 / Published online: 15 November 2003

C) Springer-Verlag 2003

\begin{abstract}
The 'attentional blink' (AB) reflects a limitation in the ability to identify multiple items in a stream of rapidly presented information. Repetitive transcranial magnetic stimulation (rTMS), applied to a site over the right posterior parietal cortex, reduced the magnitude of the $\mathrm{AB}$ to visual stimuli, whilst no effect of rTMS was found when stimulation took place at a control site. The data confirm that the posterior parietal cortex may play a critical role in temporal as well as spatial aspects of visual attention.
\end{abstract}

Keywords Transcranial magnetic stimulation - Right parietal cortex $\cdot$ Attentional blink $\cdot$ Right posterior parietal cortex $\cdot$ Visual stimuli $\cdot$ Posterior parietal cortex

\section{Introduction}

The attentional blink $(\mathrm{AB})$ is a failure of the visual system to consciously process two targets in quick succession, when masked by intervening distractors (e.g. Raymond et al. 1992, 1995). An AB can be induced in a task where participants are shown a rapid stream of letters and have to discriminate the presence of a particular target letter (e.g. an $\mathrm{X}$ ). In a baseline condition, this is the only task performed. In a second 'blink' condition, a first target (T1) is presented prior to the second target (T2 - the $\mathrm{X}$ in this instance). When T2 follows T1, then T2 discrimination is impaired - a drop in performance that can last for up to $500 \mathrm{~ms}$. Several explanations have been offered for this

A. C. G. Cooper · G. W. Humphreys $(\bowtie) \cdot$ J. Hulleman ·

P. Praamstra

Behavioural Brain Sciences, School of Psychology, University

of Birmingham,

Birmingham, B15 2TT, UK

e-mail: g.w.humphreys@bham.ac.uk

Tel.: +44-121-4144930

M. Georgeson

Neurosciences Institute, Aston University,

Aston,

Birmingham, B4 7ET, UK effect, including that it reflects: (1) the time taken to encode T1 into visual short-term memory (VSTM) (e.g. Jolicoeur et al. 2002), (2) competition for response retrieval between T1 and T2 in VSTM (Isaak et al. 1999), and (3) reduced attention to T2 (Shapiro et al. 1997). In the present study, we are less concerned with the functional mechanisms that determine the $\mathrm{AB}$, and more with evaluating the neural substrates of any effect. It is to prior studies on this topic that we now turn.

Husain and colleagues (Husain et al. 1997) found that patients with lesions to either right inferior parietal or right inferior frontal areas showed an extended $\mathrm{AB}$ (lasting much longer than normal). This was correlated with clinical measures of unilateral neglect, and taken to indicate a non-lateralised deficit in attention or VSTM encoding in these patients. Shapiro et al. (2002) extended this result by distinguishing between patients with lesions of the inferior parietal lobe/superior temporal gyrus and patients with more superior parietal regions, arguing that an extended $\mathrm{AB}$ was linked to damage to the more inferior sites. fMRI data on the effect has been reported by Marois et al. (2000). Supporting the Husain et al. study, they found activations in the intraparietal sulcus (bilaterally) and inferior, lateral right frontal areas associated with the AB. These studies suggest that processes involved in consolidating stimuli in VSTM, in modulating response selection between competing targets, and/or in allocating attention to rapidly presented visual stimuli are controlled by a frontoparietal network. This supports work by Wojciulik and Kanwisher (1999), who found overlapping activation in the intraparietal sulcus (IPs) when both spatial and non-spatial selection was called upon in tasks.

In this study, we provide converging evidence for a role of the right parietal cortex in non-spatial (time-based) visual selection, by demonstrating that transcranial magnetic stimulation (TMS) applied to this brain region selectively modulates the AB. TMS involves the application of a transient magnetic field to a selective brain region, to selectively modify, temporarily, the neuronal activity. Often the effects of TMS are characterised as causing a 'virtual lesion' that compromises performance in 
a certain task, e.g. reaction times (RTs) to targets are slowed (e.g. Ashbridge et al. 1997) or targets become difficult to detect (Corthout et al. 1999). However, TMS has also been found to enhance performance (Grosbas and Paus 2002; Hilgetag et al. 2001; Seyal et al. 1997), perhaps by increasing cortical excitability (see Grosbas and Paus 2002). In either case, the technique provides important evidence over and above that possible through functional imaging, since it indicates a causative role of a particular neural area in modulating behaviour. Furthermore, since its effects can be more localised than the effects of naturally occurring brain lesions, it can also provide more accurate information on neural localisation of function. We report data on the effects on the $A B$ of repetitive TSM (rTMS) applied over the right posterior parietal cortex (electrode site $\mathrm{P} 4$, covering a region including the superior parietal lobe (SPL) and the intraparietal sulcus (IPS); Homan et al. 1987), relative to a control condition in which TMS was applied to a posterior site over the interhemispheric fissure (electrode site Pz). Ashbridge et al. (1997) have demonstrated that TMS applied to the posterior parietal region can selectively disrupt serial visual search, indicating a role for this region in spatial selection tasks. We show modulation of the AB only when the IPS is stimulated, consistent with this brain region being involved also in non-spatial forms of visual selection (cf. Wojciulik and Kanwisher 1999). Stimulation was performed over the right $\mathrm{P} 4$ site only, to match the neuropsychological data on the AB effect (Husain et al. 1997).

\section{General method}

For all the following experiments the same basic $\mathrm{AB}$ paradigm was used, and the timing of the TMS was constant.

\section{Equipment}

The visual stimuli were presented on a $15 ", 1,078 \times 764$ resolution colour monitor set centrally $55 \mathrm{~cm}$ from the participant, connected to an Apple Macintosh iBook. The experiment was generated and controlled with Psyscope (Cohen et al. 1993) software in combination with a Carnegie-Mellon University Button Box designed to give millisecond accuracy with Psyscope. For added accuracy in the positioning of the coil, Brainsight Frameless stereotaxy equipment was employed. This equipment allows exact positioning of the coil and online monitoring of coil position with infra-red markers attached to the subjects head (via a headband) and the TMS coil. The target sites were determined by the use of an electrode cap, marked with the electrode sites according to the 10/20 system (American Electroencephalographic Society 1991).

\section{TMS procedure}

TMS was performed with a Magstim Rapid stimulator, using a 50$\mathrm{mm}$ figure-of-eight shaped coil. The TMS stimulation comprised three pulses at $125-\mathrm{ms}$ intervals $(8 \mathrm{~Hz}$ rTMS over an approximate 251-ms interval). The intensity of the TMS was set at $90 \%$ of each participant's motor threshold (see "Procedure" below). Prior to commencing the experiments the motor threshold for each participant was established. This entailed single-pulse TMS on the scalp area over the right motor cortex, starting with an initial TMS output level of $50 \%$ of maximum TMS output, and increasing output in $5 \%$ steps, until a visible movement in the left hand was demonstrated. Normally the procedure required no more than 15 or 20 TMS pulses. After determining the motor threshold, the TMS coil was positioned over one of two sites determined by the 10-20 electrode system for EEG: electrode site P4 (site: 44, $-59,48$, in the space of Talairach and Tournoux 1988) or Pz (site: $0,-32,21$ in Talairach and Tournoux 1988). The setting of the coil to the site was guided via the frameless stereotaxy system. The co-ordinates for the 10-20 electrode system were converted using the Münster T2T converter (Steinsträter et al. 2002). The coil was held by an articulated arm clamp which allowed for fixation of the coil in the target position. The coil handle was angled towards the participant's nape.

\section{$\mathrm{AB}$ procedure}

\section{AB stimuli}

The visual stimuli for the $\mathrm{AB}$ task consisted of black capital letters in Helvetica font (point size 48) presented centrally on a medium grey background. The first target letter (T1), by contrast, was white. T1 was chosen randomly from a subset of letters: N, Z, B, E, L, T, $\mathrm{W}$ and $\mathrm{M}$. The remaining letters of the alphabet, save for $\mathrm{X}$, were used as distractor letters. $\mathrm{X}$ was retained as the second target letter (T2).

\section{Procedure}

Each trial commenced with a central fixation cross (a ' + ' in Helvetica font, point size 48) presented for 1,000 ms. Immediately following the offset of the cross, a stream of black capital letters was presented at the centre of the screen. Each letter was presented for $20 \mathrm{~ms}$, followed by a blank screen for $80 \mathrm{~ms}$ (i.e. a rate of 10 letters/ s). In total 17 letters were presented per stream (total trial duration $=$ $2,700 \mathrm{~ms} ; 1,000 \mathrm{~ms}$ fixation $+1,700 \mathrm{~ms}$ for the letters). In each stream, one letter was white, forming the first target (T1). T1 could only appear in positions $4,5,6,7$ or 8 in the stream, in a randomly assigned fashion. T2 was a black capital $\mathrm{X}$, presented in the stream at some point after T1 had disappeared. The 'lag' positions for T2 were: the very next position after T1 (Lag 1), 2 positions later (Lag 2), or positions 3, 4, 5, 7 or 9 (see Fig. 1). In the experimental condition, participants were instructed to make two key presses corresponding respectively to the identity of the white letter (pressing the key corresponding to the letter), then ' $\mathrm{Y}$ ' or ' $\mathrm{N}$ ' for 'yes I did see' or 'no I didn't see' T2. In the baseline control condition, only detection of $\mathrm{T} 2$ was required. Responses were untimed, making the intertrial interval variable. There was a period of $500 \mathrm{~ms}$ following the response before the onset of the next fixation cross.

During trials in which rTMS was applied, the rTMS train was initiated directly after the offset of T1. Given the profile of the train (three pulses $125 \mathrm{~ms}$ apart), T2 s at Lags 1 or 2 fell within the duration of the train.

Prior to the experimental trial commencing, participants were given a set of practice trials with and without rTMS. During these practice trials, the experimenter monitored the eyes of the participants and the full experiment only proceeded if the participant did not show eye blinks across at least six consecutive practice trials. One participant was discarded for this reason. A second participant was discarded because eye blinks were made across experimental trials. 


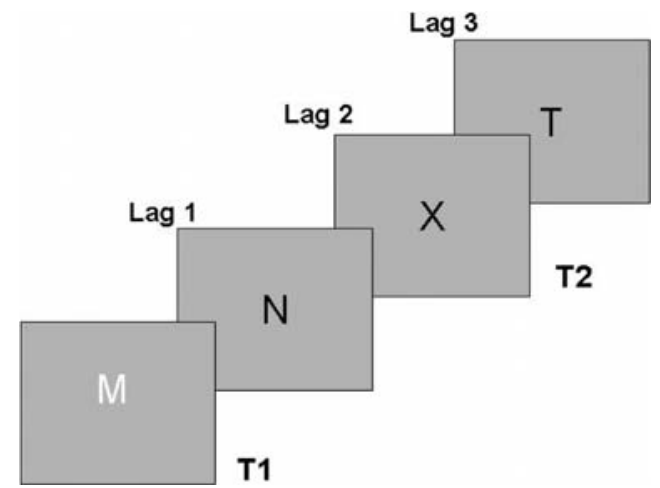

Fig. 1 Example letter sequence from the AB procedure (T2 shown at Lag 2)

Design

The $\mathrm{AB}$ experiment comprised 280 trials split into 4 blocks of 70 with a short rest between each block. The 280 trials contained 140 in which T2 was present and 140 in which it was absent. These 140 trials comprised 70 where rTMS was applied and 70 where no rTMS was applied. In the 140 trials where T2 was present, it was presented 20 times at each of the 7 sampled Lag positions following T1. The rTMS and non-TMS trials and Lag positions of T2 were mixed randomly within blocks.

Each experiment comprised three experimental factors: TMS (levels: present and absent), T2 (levels: present and absent) and Lag (levels: Lag 1, Lag 2, Lag 3-only these three were analysed since this is where any effect of interest should appear; at these lags also ceiling effects are avoided). Across the experiments, TMS site also served as a factor (levels: electrode site $\mathrm{P} 4, \mathrm{Pz}$ ). The order of the T2 only (baseline) and T1 plus T2 report blocks was counterbalanced across participants.

\section{Experiment 1: establishing TMS effects on the AB at electrode site P4}

Method

\section{Participants}

Eighteen participants were recruited (12 male, 6 female, all right handed, with an age range between 22 and 46 years). All were naive with respect to the possible effects TMS may have on the AB. Eight were right-handed and five were female. All had normal or corrected-to-normal vision. Two were paid to participate, at a rate of $£ 10 / \mathrm{h}$. Six participants were ultimately excluded, two due to eye blinks (see above) and four because they showed no AB (they were at ceiling on non rTMS trials at T2 detection at all Lags). This resulted in 12 participants producing data for the analysis. All the participants gave their written informed consent. We conformed with the Declaration of Helsinki, and formal ethics approval for the study was provided by the Ethics Committee of the School of Psychology, University of Birmingham.

\section{Procedure}

The coil was positioned over electrode site P4. In addition to the standard $\mathrm{AB}$ trials, where participants had the dual task of identifying T1 and detecting T2, we also ran 6 of the 12 participants on a separate block of trials where they performed the standard control task for the $\mathrm{AB}$, where they had only to detect $\mathrm{T} 2$. The data for these trials (carried out in a separate block with the remaining procedure identical to this experiment) are visible in Fig. 2.

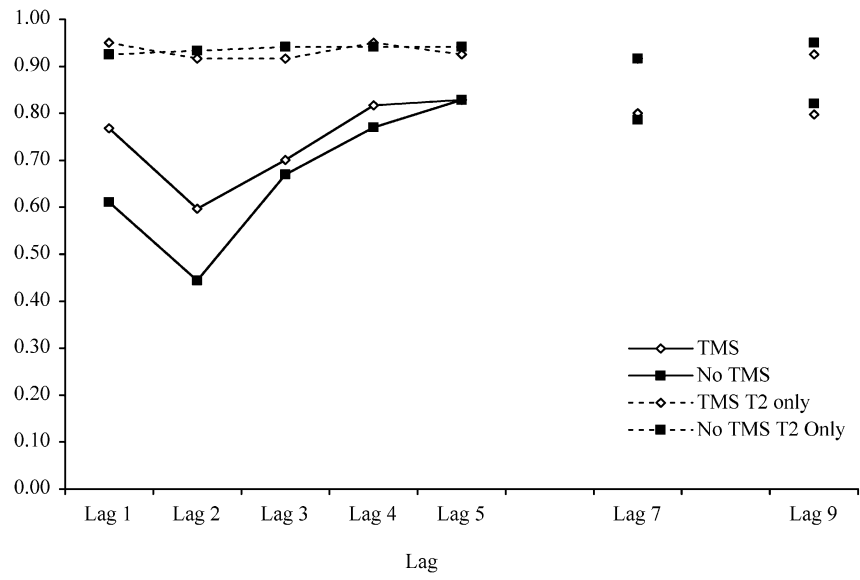

Fig. 2 Percentage correct reports of T2 with and without TMS applied to site P4 (Experiment 1)

\section{Results}

Detection performance was defined as the percentage of correct responses taken over trials with $\mathrm{T} 2$ present. The effects of administering rTMS over electrode site P4 during the $A B$ task can be seen alongside the $A B$ performance without rTMS in Fig. 2, for all Lags. A clear effect of TMS is visible, particularly at Lags 1 and 2, where detection performance on T2 improved by $16 \%$ in both Lags (from $61 \%$ to $77 \%$ in Lag 1 and from $44 \%$ to $60 \%$ in Lag 2). By Lags 3 and 4 this benefit almost disappeared, with an effect of only $3 \%$ and $5 \%$ respectively visible. A two-way ANOVA with TMS (present vs absent) and Lag (Lag 1, Lag 2, and Lag 3) revealed a strong main effect of TMS $\left(F_{(1,11)}=15.4\right.$, $p=0.002)$ and of $\operatorname{Lag}\left(F_{(1,11)}=3.64, p=0.04\right)$. The interaction was not reliable. Over these three Lags, TMS improved detection performance by $9 \%$ on average. With planned contrasts of rTMS vs no TMS means at each Lag, the TMS effect was shown to be due to improved performance at Lags $1 \quad(p=0.005)$ and 2 $(p=0.005)$, but not at Lag $3(p=0.53)$.

An analysis of T1 accuracy between the two TMS conditions, using a paired $t$-test on percentage accuracy of T1, showed T1 accuracy was not worse when rTMS was present (mean accuracy: 97\% correct) relative to when it was absent (mean accuracy: 96\%; $t_{(11)}=1.21, p=0.25$ ). There were no effects of TMS on false alarm rates (responding that $\mathrm{T} 2$ was present when it was absent; with rTMS, the mean false alarm rate was $3.9 \%$; without rTMS it was $3.0 \%$; $\left.t_{(11)}=-0.69, p=0.50\right)$.

No effects of TMS on the AB control task (detect T2 only) were visible, as can be seen in Fig. 2.

\section{Discussion}

The application of rTMS over electrode site P4 produced a clear $16 \%$ benefit in $\mathrm{T} 2$ detection rates at Lags 1 and 2 in the $\mathrm{AB}$. This benefit may be attributed either to a facilitatory effect of rTMS on T2 or a suppressive effect 
on T1. For example, the $\mathrm{AB}$ may be reduced if rTMS speeds the consolidation of T1 in VSTM or if it boosts the allocation of attention to T2. It may also be that, by disrupting activation from $\mathrm{T} 1$, there is reduced competition from T1 for response selection to T2 (cf. Isaak et al. 1999). Note, however, that any disruptive effect of rTMS on T1 was not sufficient to make T1 harder to identify. It is also possible though that the beneficial effect of rTMS here was due to increased generalised arousal, directly from the TMS itself or from the auditory 'click' made when the TMS pulse was emitted. To test this, Experiment 2 repeated the study but applied TMS to a control site overlying the interhemispheric fissure, rather than cortical tissue. If general arousal alone were important, similar effects should emerge here.

\section{Experiment 2: effects on the $A B$ from stimulating over electrode site $\mathbf{P z}$}

Method

\section{Participants}

Fifteen participants (13 of whom had been run in Experiment 1) took part ( 9 males, 6 females, all right handed, with ages between 22 and 46 years). Eight carried out Experiment 2 before they took part in Experiment 1. Three participants were ultimately excluded, two because they showed no AB and one who made eye blinks. This left 12 participants all of whom had taken part in both experiments. Five took part in this experiment first; the remaining seven performed Experiment 1 first.

\section{Procedure}

The procedure was identical to that described in the "General method." The coil was positioned over electrode site Pz.

Results

No discernable effects of rTMS were observed. A twoway ANOVA comparing rTMS (presence vs absence) and Lag (Lags 1-3) showed only a main effect of Lag $\left(F_{(1,11)}=7.31, p=0.004\right)$ with no other main effects or interactions. At best there was only an unreliable $2 \%$ mean increase in T2 detection over Lags 1-3 from applying rTMS to the region under $\mathrm{Pz}$ (detection accuracy with no rTMS: 58\%; with rTMS: 60\%) (see Fig. 3). Planned comparisons of accuracy means for rTMS vs no rTMS for Lags 1-3 did not reveal any reliable differences. There were no effects of TMS on false alarm rates $(F<1.0)$.

\section{Combined analysis of $\mathrm{P} 4$ and $\mathrm{Pz}$ data}

To test effects across stimulation sites, we conducted a three-way within-subjects repeated measures ANOVA with the factors being TMS (present vs absent), Lag (Lags 1 and 2) and site (P4 vs Pz). Main effects of TMS

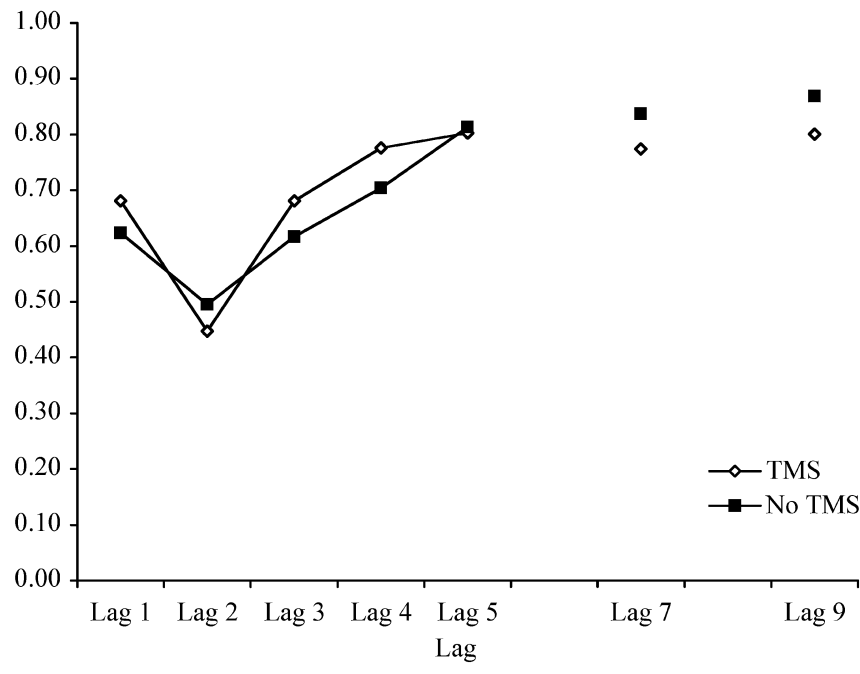

Fig. 3 Percentage correct reports of T2 with and without TMS applied to location $\mathrm{Pz}$ (Experiment 2)

(mean accuracy rTMS absent: 54\%, rTMS present: 62\%; $\left.F_{(1,11)}=17.66, p<0.001\right)$ and $\operatorname{Lag}\left(F_{(2,22)}=11.13, p=0.007\right)$ were observed, as well as an interaction between TMS and site $\left(F_{(1,11)}=7.31, p<0.025\right)$. There were no other interactions.

Planned comparisons of means showed that the presence of rTMS on P4 significantly improved performance over the no TMS condition $(p=0.003)$. Stimulating over P4 also significantly improved performance over and above stimulating at site $\mathrm{Pz}$ (over Pz: $60 \%$, over P4, 69\%, $p=0.014$ ). No reliable differences were found for the noTMS trials with the coil positioned over $\mathrm{P} 4$ or Pz.

The difference in performance between the TMS and no-TMS conditions, with TMS applied either to P4 or to $\mathrm{Pz}$, can be seen in Fig. 4.

\section{Discussion}

It is clear that stimulating over electrode site Pz produced no reliable effects on the $\mathrm{AB}$. The improvement in the $\mathrm{AB}$, found when rTMS was applied over site P4, is unlikely to be due to general arousal.

\section{General discussion}

In Experiment 1 we found that stimulating with $8 \mathrm{~Hz}$ rTMS at $90 \%$ of motor threshold at electrode site P4 (right parietal cortex), immediately following the offset of T1, reliably improved the detection of T2 by $16 \%$ at Lags 1 and 2 only. There was no effect on the report of T1 itself, and no effect on report of $\mathrm{T} 2$ in the control condition (without report of T1). In Experiment 2 we failed to find a similar effect from rTMS applied to a site under $\mathrm{Pz}$, countering an argument that the earlier benefits were caused by the stimulation and/or the associated auditory clicks increasing arousal. 
Fig. 4 Differences between T2 detection with and without TMS, applied either to location $\mathrm{P} 4$ or to $\mathrm{Pz}$ (a positive score indicates better detection of $\mathrm{T} 2$ under TMS)

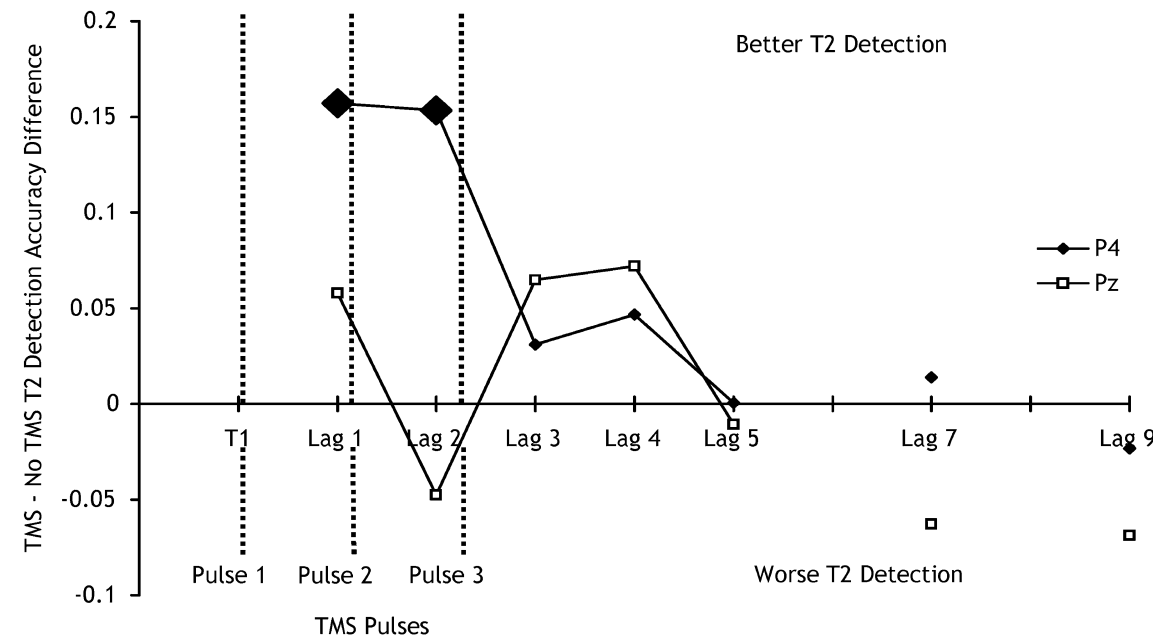

There are several ways in which rTMS at site P4 could improve the AB. TMS could have a facilitatory effect because it operated in an excitatory manner to either: (1) reduce the time taken to encode T1 into VSTM (e.g. by benefiting temporal segmentation); or (2) generate enhanced temporal attention to T2. Alternatively, if TMS had an inhibitory influence, the $\mathrm{AB}$ could have been reduced because stimulation lessened response competition from T1, improving T2 report (Isaak et al. 1999). The present data do not discriminate between these alternative views. Nevertheless, the data do implicate the right posterior parietal cortex (and in particular the superior region of the intraparietal sulcus) in non-spatial forms of visual selection - in this case the selection of visual stimuli over time. This fits with data from brain imaging demonstrating activation of this region in a range of visual selection tasks, non-spatial as well as spatial (Wojciulik and Kanwisher 1999). For example, in one recent imaging study, Pollmann et al. (2003) examined the brain regions activated when participants used time differences between visual displays to help segment irrelevant from relevant stimuli in visual search. They found early activation particularly in the right superior parietal/precuneus region, the right intraparietal area and the posterior superior temporal sulcus when temporal segmentation led to efficient search. They suggest that the intraparietal sulcus and regions superior to it play a role in temporal aspects of visual selection, whilst more interior regions may be involved in responding to the results of any temporal segmentation process. The extended $\mathrm{AB}$ found in patients with inferior parietal regions, then, may be caused by a failure to register stimuli that are temporarily segmented under RSVP conditions (Shapiro et al. 2002). In contrast, the P4 site stimulated in the present study overlaps relatively superior regions of the intraparietal sulcus (Homan et al. 1987), so that activation here could reflect changes in the segmentation process itself, rather than detecting the products of segmentation. An intriguing question for future research then, is whether different components of the $\mathrm{AB}$ may be separated by stimulation of different brain regions. It is also the case that bilateral rather than purely unilateral activity has been found in imaging studies of the $A B$ (Marois et al. 2000) and temporal segmentation (Pollmann et al. 2003), and it is an open question as to whether activation of a left hemisphere homologue to the right hemisphere site stimulated here would similarly modulate the blink effect.

As noted in the "Introduction", TMS has often been used to produce 'virtual lesions', generating temporary disruptions to tasks (Walsh and Cowey 1998), though some performance enhancing effects have been previously reported (Grosbas and Paus 2002; Oliveri et al. 2001). Grosbas and Paus speculated that facilitatory effects on visual spatial detection after TMS on the frontal eye field might be mediated by a TMS-induced increase of cortical excitability. Possibly the beneficial effects we have found are likewise attributable to increased excitability, in our case of parietal cortex. Increased excitability of the parietal cortex as the underlying mechanism might facilitate disengagement of attention from $\mathrm{T} 1$ and thereby speed up its application to T2 (see above). Note however that there is at least one 'suppressive' account of the TMS effects, on T1, that would also generate the observed pattern of data. We used a short repetitive $8-\mathrm{Hz}$ protocol, with stimulation below motor threshold. Some studies in the motor system suggest that high-frequency (above $1 \mathrm{~Hz}$ ) rTMS tends to exert excitatory rather than inhibitory effects on neuronal firing (Gangitano et al. 2002), giving some support for an increased excitability induced by our protocol. But unlike stimulation of the motor cortex or the visual cortex, where increases or decreases in excitability can be assessed by means of motor responses or phosphene thresholds, parietal cortex stimulation does not allow straightforward inferences regarding the direction of excitability changes induced by TMS. Clearly, to help interpret the neuronal mechanisms underlying the behavioural effects induced by TMS, further work is needed to assess whether particular protocols that reduce or enhance cortical excitability in the motor cortex have the same effect when applied at different brain regions.

Acknowledgements This work was supported by a grant from the Medical Research Council (UK). 


\section{References}

American Electroencephalographic Society (1991) American Electroencephalographic Society guidelines for standard electrode position nomenclature. J Clin Neurophysiol 8:200-202

Ashbridge E, Walsh V, Cowey A (1997) Temporal aspects of visual search studies by transcranial magnetic stimulation. Neuropsychologia 35:1121-1131

Cohen JD, MacWhinney B, Flatt M, Provost J (1993) PsyScope: a new graphic interactive environment for designing psychology experiments. Behav Res Methods Instrum Comput 25:257-271

Corthout E, Uttl B, Ziemann U, Cowey A, Hallett M (1999) Two periods of processing in the (circum)striate visual cortex as revealed by transcranial magnetic stimulation. Neuropsychologia $37: 137-145$

Gangitano M, Valero-Cabre A, Tormos JM, Mottaghy FM, Romero JR, Pascal-Leone A (2002) Modulation of input-output curves by low and high frequency repetitive transcranial magnetic stimulation of the motor cortex. Clin Neurophysiol 113:12491257

Grosbras M-H, Paus T (2002) Transcranial magnetic stimulation of the human frontal eye field: effects on visual perception and attention. J Cog Neurosci 14:1109-1120

Hilgetag CC, Théoet H, Pascual-Leone A (2001) Enhanced visual spatial attention ipsilateral to rTMS-induced 'virtual' lesions of human parietal cortex. Nature Neurosci 3:284-291

Homan RW, Herman J, Purdy P (1987) Cerebral location of international 10-20 system electrode placement. Electroencephalogr Clin Neurophysiol 66:376-382

Husain M, Shapiro KL, Martin J, Kennard C (1997) Abnormal temporal dynamics of visual attention in spatial neglect patients. Nature 385:154-156

Isaak MI, Shapiro KL, Martin J (1999) The attentional blink reflects retrieval competition among multiple rapid serial visual presentation items: test of an interference model. J Exp Psychol Hum Percept Perform 25:1774-1792
Jolicoeur P, Tombu M, Oriet C, Stevanovski B (2002) From perception to action: making the connection-a tutorial. In: Prinz W, Hommel B (eds) Common mechanisms in perception and action: attention and performance XIX. Academic Press, London, pp 558-586

Marois R, Chun MM, Gore JC (2000) Neural correlates of the attentional blink. Neuron 28:299-308

Oliveri M, Bisiach E, Brighina F, Piazza A, La Bua D, Fierro B (2001) rTMS of the unaffected hemisphere transiently reduces contralesional visuospatial neglect. Neurology 57:1338-1340

Pollmann S, Weidner R, Humphreys GW, Olivers CNL, Müller K, Lohmann G, Wiggins CJ, Watson DG (2003) Separating segmentation and target detection in posterior parietal cortexan event-related fMRI study of visual marking. Neuroimage 18:310-323

Raymond JE, Shapiro KL, Arnell KM (1992) Temporary suppression of visual processing in an RSVP task: an attentional blink? J Exp Psychol Hum Percept Perform 18:849-860

Raymond JE, Shapiro KL, Arnell KM (1995) Similarity determines the attentional blink. J Exp Psychol Hum Percept Perform 21:653-662

Seyal M, Siddiqui I, Hundal NS (1997) Suppression of spatial localization of a cutaneous stimulus following transcranial magnetic pulse stimulation of the sensorimotor cortex. Electroencephalogr Clin Neurophysiol 105:24-28

Shapiro K, Arnell KM, Raymond JE (1997) The attentional blink. Trends Cog Sci 1:291-296

Shapiro K, Hillstron A, Husain M (2002) Control of visuotemporal attention by inferior parietal and superior temporal cortex. Curr Biol 12:1320-1325

Steinsträter O, Sommer J, Deppe M, Knecht S (2002) The Münster T2T-converter. Available at: http://neurologie.uni-muenster.de/ ger/t2tconv/

Talairach J, Tournoux P (1988) Co-planar stereotaxic atlas of the human brain. Thieme Medical, New York

Walsh V, Cowey A (1998) Magnetic stimulation studies of visual cognition. Trends Cog Sci 2:103-110

Wojciulik E, Kanwisher N (1999) The generality of parietal involvement in visual attention. Neuron 23:747-764 